\section{Rahmenbedingungen intramuraler Therapie von Sexualstraftätern}

\section{V} or dem Hintergrund der durch die Gesetzesreform seit Januar 1998 gewachsenen Möglichkeiten für und gestiegenen Erwartungen an die therapeutische Beeinflussung von Sexualstraftätern ${ }^{1}$ scheint es erforderlich, jenseits der dringend notwendigen Diskussion über Therapieformen und deren Evaluation sich auch über die Rahmenbedingungen derartiger Therapieangebote und speziell über deren Umgang mit der Schweigepflicht zu verständigen.

Im folgenden soll es um Therapieangebote im Regelvollzug innerhalb der JVA gehen, da zu erwarten ist, dass nicht alle Täter die im StVollzG benannten Voraussetzungen für die Aufnahme in die Sozialtherapie erfüllen werden und überdies zu vermuten ist, dass diese Einrichtungen auch kapazitätsmäßig nicht in der Lage sein werden, alle infrage kommenden Delinquenten aufzunehmen. Therapie in der JVA wird somit subsidiär für jene in einer Sozialtherapeutischen Anstalt angeboten werden müssen (Rotthaus 1998).

Ganz allgemein besteht im Regelvollzug die Tendenz, auf Erkenntnisse aus der psychotherapeutischen Behandlung zurückzugreifen: Bekanntlich verlangt $§ 182$, Abs. 2 und Abs. 4 StVollzG (in der Fassung vom 26.8.1998) von den Schweigepflichtsbewahrern (gem. § 203 StGB Abs. 1, 2 und 5 also auch Ärzten, Berufspsychologen und Sozialarbeitern), »sich gegenüber dem Anstaltsleiter zu offenbaren, soweit dies für die Aufgabenerfüllung der Vollzugsbehörde oder zur Abwehr von erheblichen Gefahren für Leib oder Leben des Gefangenen oder Dritter erforderlich ist « (wobei dies für Ärzte nur als Befugniserteilung und nicht als Auflage formuliert ist). Ver- stärkt wird dieses Bestreben, auf Inhalte der Psychotherapie zuzugreifen, durch die gem. § 454 Abs. 2 StPO, nun beinahe obligate Prognosebegutachtung zur Prüfung des Antrags auf Strafrestaussetzung zur Bewährung (sog. 2/3-Entlassung) und auch - wenngleich sich dies zahlenmäßig noch nicht abzeichnet durch die neuen gesetzlichen Regelungen zur vorbehaltlich angeordneten Sicherungsverwahrung gem. § 66a StGB: Die Entscheidung über die Anordnung oder Nicht-Anordnung der vorbehaltlich angeordneten Sicherungsverwahrung bedarf gem. § 275a StPO auch der externen Begutachtung.

Es gilt also, zwischen den berechtigten Sicherheitsinteressen der Allgemeinheit (vertreten durch die JVA) einerseits und dem notwendigen therapeutischen Schutzrahmen andererseits zu vermitteln. Zunächst ist nachvollziehbar, dass Anstalts-
Hartmut A.G. Bosinski

Hoffnung, der Therapeut möge »für ihn gut sagen «. In einer solchen Pseudo-Therapie würden gerade nicht die problemhaften Anteile der Persönlichkeit thematisiert, sie würde vielmehr Fassadenpersönlichkeiten favorisieren.

\section{Das Kieler Behandlungsmodell}

Seit Mitte der 80er Jahre wird durch das schleswig-holsteinische Justizministerium ein an der Kieler Sexualmedizinischen Forschungs- und Beratungstelle angesiedeltes Drittmittelprojekt zur »Intramuralen Therapie von Sexualstraftätern« finanziert. Gegenwärtig arbeiten zwei Psychologen unter fachlicher Anleitung und Supervision des Autors in diesem Projekt in zwei Justizvollzugsanstalten des Landes. Tabelle 1 zeigt die Behandlungszahlen der Jahre 1999-2001.

Andererseits: Sobald Einblicke in individuelle Therapieinhalte genommen werden, ist dies ein Einbruch in den notwendig geschützten Rahmen der Psychotherapie, der letztlich kontraproduktiv zu einem der intendierten Ziele der Reform größere Sicherheit der Allgemeinheit durch Besserung der Täter wäre. Der Patient, der sich nicht der Verschwiegenheit seiner Äußerungen sicher sein kann, wird keinen Einblick in seine tatsächlichen Gedanken, Gefühle, Motive, Neigungen und Impulse geben, er wird vielmehr bemüht sein, »einen guten Eindruck zu machen«, immer in der
Die therapeutische Arbeit folgt einem kognitiv-behavioralen Ansatz, der hier nur hinsichtlich seines Ablaufs und der Rahmenbedingungen skizziert werden soll:

Jeder Strafgefangene mit einem Sexualdelikt wird bei seiner Aufnahme in die JVA auf die Möglichkeit einer intramuralen Therapie (mit Name und Sprechzeiten des Therapeuten) hingewiesen. Es wird ihm nahegelegt, an den Therapeuten ei-
Tabelle 1: Behandlungszahlen im Rahmen des Kieler Forschungsprojektes »Intramurale Therapie von Sexualstraftätern«

\begin{tabular}{|c|c|c|c|c|}
\hline & $\mathbf{1 9 9 9}$ & $\mathbf{2 0 0 0}$ & $\mathbf{2 0 0 1}$ & $\mathbf{2 0 0 2}$ \\
\hline $\begin{array}{l}\text { Behandelte Täter mit Delikten gem. § 176 StGB } \\
\text { (Sexueller Kindesmissbrauch) }\end{array}$ & $\mathbf{2 8}$ & $\mathbf{3 6}$ & $\mathbf{3 9}$ & $\mathbf{1 8}$ \\
\hline Aufschub/ Abbruch der Behandlung & 13 & 11 & 24 & 9 \\
\hline Wegen Leugnens & 9 & 9 & 12 & 5 \\
\hline Wegen mangelnder Kooperativität o.ä. & 2 & 2 & 10 & 3 \\
\hline Wegen mangelnder Sprachkenntnisse & 2 & - & 2 & 1 \\
\hline $\begin{array}{l}\text { Behandelte Täter mit Delikten gem. §§ 177, 178 StGB } \\
\text { (Vergewaltigung/ sex. Nötigung) }\end{array}$ & $\mathbf{2 3}$ & $\mathbf{2 0}$ & $\mathbf{2 8}$ & $\mathbf{2 3}$ \\
\hline Aufschub/ Abbruch der Behandlung & 13 & 9 & 9 & 11 \\
\hline Wegen Leugnens & 9 & 7 & 7 & 7 \\
\hline Wegen mangelnder Kooperativität o.ä. & 4 & 2 & 2 & 4 \\
\hline Sonstige behandelte Sexualstraftäter & $\mathbf{1}$ & $\mathbf{1}$ & $\mathbf{2}$ & $\mathbf{1}$ \\
\hline
\end{tabular}

Quelle: Bosinski et al. (2003) SEXUOLOGIE 9: 39-47 
Abbildung 1: Übersicht über die Ebenen des Therapiekonzeptes

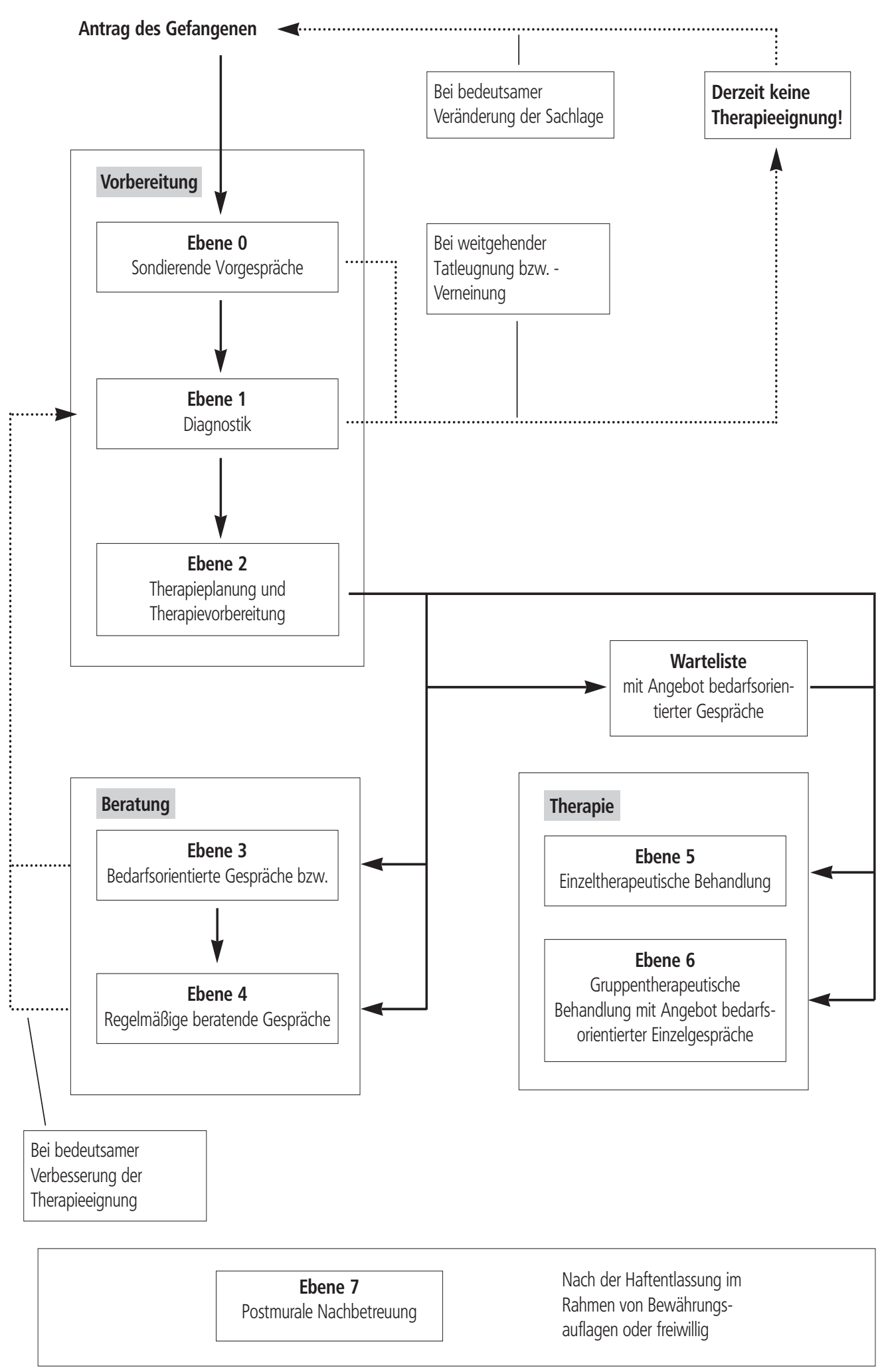

nen Antrag auf ein erstes Gespräch zu stellen.

Die Betreuung ist in drei Komplexe (Vorbereitung, Beratung, Therapie) gegliedert, innerhalb derer verschiedene Ebenen unterschieden werden, die wiederum in Phasen $(\mathrm{a}=$ Phase der Klärung, $b=$ Phase der Weiterentwicklung) unterteilt sind (s. Abbildung 1).

Alle Vollzugsbeamten (und auf Wunsch auch die Prognosegutachter) erhalten das differenzierte Therapiekonzept mit einer schriftlichen Handreichung, in der verdeutlicht wird, dass zwar die numerische Reihenfolge der Ebenen nicht unbedingt eine notwendige Abfolge darstellen, die jeder Patient zur Erreichung der angestrebten Therapieziele zwangsläufig durchlaufen muß, dass aber das Aufsteigen in eine »höhere Ebene« als ein gewisser Therapiefortschritt gewertet werden kann. Sie werden darüber informiert, dass für den Wechsel einer Ebene bestimmte Voraussetzungen erfüllt sein müssen, dass, wenn diese nicht gegeben sind, die Ebene bis auf weiteres beibehalten (die Therapie also stagniert), und dass die Behandlung erforderlichenfalls auch unterbrochen bzw. vollständig abgebrochen werden kann.

\section{Der Umgang mit der Schweigepflicht}

Das von uns erarbeitete Procedere zum Umgang mit der Schweigepflicht orientiert sich an den sog. Sankelmarker Thesen (Beier \& Hinrichs, 1995) und sieht eine standardisierte Form der Meldung über den Stand der Therapie an die Anstaltsleitung oder an den mit der Prognose zum 2/3-Termin beauftragten Gutachter vor (s. Abbildung 2):

Da die Mitarbeiter der JVA und ggf. auch der Prognosegutachter das oben referierte Therapiekonzept kennen, können sie relativ rasch erkennen, in welchem Abschnitt der Betreuung sich der betreffende Gefangene befindet, ob in der Therapie-Vorbereitung (Ebene 0,1 oder 2 ), auf der Beratungsebene (Ebene 3 und 4) oder in der Einzel- bzw. Gruppentherapie (Ebene 5 bzw. 6, jeweils differenziert nach Phase der Klärung oder Phase der Weiterentwicklung). 
Ebenso ist erkennbar, wann, durch wen und warum es zum Abbruch einer Therapie (etwa bei mangelnder Kooperativität) gekommen ist. Diese Informationen können in die Überlegungen $\mathrm{zu}$ Lockerungen oder vorzeitiger Entlassung miteinbezogen werden. Darüber hinaus gehende, individuelle Angaben zu den konkreten Patienten werden hingegen nicht gemacht. Es versteht sich von selbst, dass weder die Therapeuten noch ihr Supervisor für die Begutachtung der behandelten Probanden zur Verfügung stehen.

Um der Gefahr einer Instrumentalisierung der Therapie vorzubeugen, wird vor Beginn jeder Therapie mit den Patienten folgender schriftlicher Vertrag geschlossen (s. Kasten nächste Seite):

Auf diese Weise soll verhindert werden, dass der Gefangene den Therapeuten gegenüber der Strafvollstreckungskammer von der Schweigepflicht entbindet, wo er dann - als sachverständiger Zeuge - Aussagen zum individuellen Verlauf machen müsste (womit eben das Therapieziel konterkariert würde). Diese Vereinbarung hat rechtlich zwar nur bedingt bindenden Charakter, hat sich jedoch bislang auch deshalb bewährt, weil für alle Beteiligten erkennbar wird, dass ein Patient, der sie bricht, nicht wirklich die Therapie wünscht (da er damit deren Abbruch initiiert), sondern diese für Lockerungen oder vorzeitige Haftentlassung instrumentalisiert.

\section{Grenzen}

Zwar hat sich die oben dargestellte Praxis, deren Konformität mit dem neugefassten § 182 StVollzG und durch das Justizministerium des Landes Schleswig-Holstein ausdrücklich bestätigt wurde, nun bereits über Jahre bewährt. Aufgrund dieser jahrelangen Vorarbeit kann heute in Schleswig-Holstein jedem inhaftierten Sexualstraftäter eine Therapie angeboten werden.

Gleichwohl stößt die Psychotherapie bei strafgefangenen Sexualstraftätern im Regelvollzug, verglichen mit der Behandlung von auf freiem Fuß befindlichen Probanden in einer frei zugänglichen Ambulanz, immer auf eine Reihe systemimmanenter Grenzen. Einige wer-
Abbildung 2: Formular zur Therapie

Therapieinformation

Datum:

\begin{tabular}{|lccc}
\hline & & \\
\\
Name, Vorname & Gefg.B.Nr. & Geburtsdatum \\
& & \\
\hline
\end{tabular}

Beginn der Behandlung

Der Patient hat seit dem

insgesamt ….......... Termine wahrgenommen.
Ende der Behandlung

Die Behandlung wurde am

durch den

\section{$\square$ Therapeuten $\square$ Patienten unterbrochen $\square$ beendet. $\square$ Die Behandlung dauert an.}

\section{Derzeitiger Stand der Behandlung}

૫) Sondierende Vorgespräche (0)

4 Diagnostik (1)

( Therapieplanung $\mathrm{u}$. Therapievorbereitung (2)

( Bedarfsorientierte Gespräche bzw. Krisenintervention (3)

૫ Regelmäßige beratende Gespräche (4)

(1) Einzeltherapeutische Behandlung (5)

(] Gruppentherapeutische Behandlung (6)

Q Phase der Klärung (a)

P Phase der Weiterentwicklung (b)

( Die wesentlichen Therapieziele konnten erreicht werden.

D Die wesentlichen Therapieziele konnten (bisher) nicht vollständig erreicht werden.

(- Ein wesentlicher Therapiefortschritt ist derzeit nicht mehr zu erwarten.

( Mangelnde Schuldeinsicht des Patienten.

4. Mangelhafte Kooperativität des Patienten.

(1) Der Patient verneint bzw. leugnet die verurteilte(n) Straftat(en).

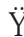

Ebenen in Klammern nach Therapiekonzept 3/1999

Die Zuordnung von Patienten zu bestimmten Betreuungsstadien stellt keine Einschätzung ihrer Eignung für Vollzugslockerungen oder vorzeitige Entlassung dar.

૫ Gegenwärtig ist kein freier Therapieplatz vorhanden. Der Patient steht auf der Warteliste.

૫ Nach der Haftentlassung ist eine regelmäßige psychotherapeutische Weiterbetreuung geboten.

\section{Anmerkungen:}

Quelle: Bosinski et al. (2003) SEXUOLOGIE 9: 39-47 
den unabänderlich sein; aus ihnen die Unmöglichkeit therapeutischer Beeinflussung von Sexualstraftätern in diesem Setting abzuleiten, käme einem therapeutischen Nihilismus gleich. Neben den strukturellen und räumlichen Einschränkungen sowie den sprachlichen Barrieren bei Nicht-Muttersprachlern seien hier vor allem folgende Probleme genannt:

1. Fehlende oder nur sehr beschränkte Möglichkeit zur Einbeziehung einer Partnerin: Neben den Einschränkungen für die Diagnostik (Validierung von Angaben zur vita sexualis) setzt dies einer Therapie, die den kommunikativen Aspekt der - prinzipiell auf ein Gegenüber angelegten - Sexualität in den Vordergrund stellt (Beier et al. 2001), enge Grenzen. Die Bearbeitung der kommunikativen Funktion des Sexuellen muss so zumeist auf der virtuellen Ebene bleiben.

2. Fehlende oder nur eingeschränkt mögliche Exposition in vivo.

3. Problem der fehlenden Tat-Anerkennung durch den Verurteilten: Ca. $30 \%$ der Gefangenen behaupten zunächst, es handele sich in ihrem Falle um ein Fehlurteil oder man habe die Taten nur auf anwaltlichen Rat eingeräumt (um ein geringeres Strafmaß zu erreichen). Zwar reduziert sich dieser Prozentsatz im Laufe der vorbereitenden bzw. der auch diesen Probanden angebotenen bedarfsorientierten und Beratungs-Gesprächen (Ebene 3 und 4), die weiterhin tatleugnenden Gefangenen können jedoch schon deshalb nicht in Therapie genommen werden, weil diese sich sonst in einer nachträglichen »Beweiswürdigung« erschöpfen würde.

4. Häufig fehlende fachkundige Begutachtung im erkennenden Verfahren: Nach unserem Eindruck werden höchstens $15 \%$ aller Sexualstraftäter im erkennenden Verfahren begutachtet. Die Gründe hierfür sind sicher vielfältig: Neben fehlender Gutachterkapazität und deshalb von den Gerichten befürchtete Verfahrensverlängerung dürfte es insbesondere die Tatsache sein, dass das Gericht oft schon aus eigener Anschauung keine Hinweise für eine erheblich verminderte oder gar aufgehobene Schuldfähigkeit sieht.
Dabei wird jedoch ausgeblendet, dass der Gutachter bei seinen Feststellungen zur Schuldfähigkeit eine Diagnose und ggfs. eine Indikation zur Behandlung stellt, somit »Anschlusstatsachen « für eine dann folgende Therapie schafft. Er hat dabei den Vorteil, nicht durch die Schweigepflicht gebunden $\mathrm{zu}$ sein und kann wesentlich mehr und bessere Erkenntnismittel einsetzen als der intramurale Psychotherapeut. Neben einer Reihe medizinisch-technischer Untersuchungsmethoden ist hier vor allem die - nach entsprechend gebotener prozessualer Absicherung - Befragung signifikanter Dritter zu nennen, die gerade in rebus sexualibis von eminenter Bedeutung ist.

Diese Einschränkung hinsichtlich fehlender Vorgutachten gilt im übrigen in vollem Umfang auch für die gerichtlich angewiesene ambulante Therapie von Sexualstraftätern im Rahmen der Bewährung oder der Führungsaufsicht gem. § 56c StGB: So beobachten wir das Kuriosum, dass Richter - die ja eine Diagnose und Therapie-Indikation nicht stellen können - eine solche Weisung zur Psychotherapie ohne sachverständige Begutachtung des Probanden erlassen. Es bleibt dabei völlig ungeprüft, ob eine Therapie überhaupt (ambulant) möglich ist. Dies geht soweit, dass Freiheitsstrafen nur deshalb zur Bewährung ausgesetzt werden, weil der Verurteilte sich bereit erklärt, eine psychotherapeutische Behandlung wahrzunehmen. Findet er keinen Therapieplatz - weil es zu wenige gibt, oder weil Therapeuten die Behandlung aus Gründen, die in der Person des Verurteilten liegen (Sprachbarriere, erhebliche intellektuelle Minderbegabung, Tatleugnung usw.) nicht übernehmen können -, so bleibt der Verurteilte (der ja der Weisung folgen will, dessen Bewährung mithin nicht widerrufen werden kann) unbehandelt in Freiheit.

Während also Begutachtungen im Erkenntnisverfahren fehlen hat die Praxis inzwischen gezeigt, dass die gem. § 454 Abs. 2 StPO, (n.F.) beinahe regelhaft veranlasste Prognose-Begutachtung zur sog. $2 / 3^{-}$ Entlassung von Sexualstraftätern mit einer zeitigen Freiheitsstrafe in einigen wenigen Fällen »zu spät kommt«. Wir hatten bereits im Vor-

\section{Therapievertrag}

Um psychotherapeutische Hilfestellung zu ermöglichen, ist der Aufbau einer offenen, ehrlichen und von gegenseitigem Vertrauen getragenen Beziehung notwendig. Dazu sollen die folgenden Punkte vertraglich geregelt werden:

\section{Schweigepflicht}

Alle Informationen, die im Rahmen der psychotherapeutischen Gespräche offenbar werden, unterliegen einer gesetzlichen Schweigepflicht und werden daher streng vertraulich behandelt. Nur im Rahmen der therapeutischen Supervision, das ist die fachliche Beratung von Therapeuten, kann ohne Namensnennung über Therapieinhalte gesprochen werden. Allgemeine Informationen über die Einhaltung der Therapietermine und Therapiephasen können an die Leitung der JVA weitergegeben werden. Eine darüber hinausgehende Entbindung von der Schweigepflicht durch den Patienten ist vertragswidrig und führt zur Beendigung der Therapie.

\section{Therapiestunden}

Therapiestunden werden in gemeinsamer Absprache vereinbart. Das bedeutet, daß sich beide Seiten an die vereinbarten Termine zu halten haben. Sollten wichtige Gründe für eine Absage vorliegen, so ist die jeweils andere Seite möglichst frühzeitig darüber zu informieren und ein neuer Termin abzusprechen.

\section{Beenden der Therapie}

Falls wichtige Faktoren einen weiteren Therapiefortschritt wesentlich behindern oder unwahrscheinlich machen, kann die Therapie von beiden Seiten jederzeit beendet werden. Eine derartige Entscheidung ist dann der jeweils anderen Seite zu erläutern. Die oben geregelte Schweigepflicht gilt jedoch auch über das Therapieende hinaus.

\section{Erklärung}

Ich habe die oben dargestellte Vereinbarung gelesen, ihren Inhalt verstanden und erkläre mich mit allen Punkten einverstanden.

Ort, Datum Unterschrift

Patient; Therapeut

feld der Strafrechtsänderung (Bosinski 1997) darauf hingewiesen, dass bei der gutachterlichen Feststellung einer nach wie vor bestehenden Gefährlichkeit zum Zeitpunkt der beantragten »vorzeitigen Entlassung « sich tatsächlich nicht viel ändert: Der Gefangene muss seine Haftzeit voll verbüßen, kommt dann aber »unverändert « in Freiheit, allerhöchstens mit Anordnung einer Führungsaufsicht und entsprechenden Weisungen (deren Nicht-Befolgung aber aus unserer Erfahrung nur geringe Konsequenzen nach sich zieht).

Diese - u.E. allerdings seltene Konstellation dürfte mit ursächlich dafür sein, dass in einigen Bundesländern die »nachträgliche Sicherungsverwahrung « diskutiert bzw. bereits eingeführt wurde. Gegen eine solche post festum Verhängung der Sicherungsverwahrung wurden von verschiedenen Seiten nachvollziehbare verfassungsrechtliche Bedenken vorgebracht. Zugleich wurde im August 2002 mit Einführung des $§ 66$ a StGB vom Gesetzgeber die Möglichkeit eröffnet, die Sicherungsverwahrung im erkennenden Verfahren vorbehaltlich anzuordnen und ihre letztliche Anordnung vom Verlauf der Regelhaftzeit abhängig zu machen. Dies erfordert dann also die zweimalige Begutachtung des Probanden, nämlich im erkennenden Verfahren und (gem. § 275a StPO) im Haftverlauf, spätestens sechs Monate vor dem 2/3Termin. Wie schon bei der Prognosebegutachtung zur 2/3-Entlassung wäre es u.E. fatal, wenn interne Er- 
kenntnisse aus der Therapie hierbei unmittelbar herangezogen würden. Das in der Kieler Sexualmedizin in Anlehnung an die Sankelmarker Thesen entwickelte und oben ausführlich referierte Verfahren hat sich in der Praxis bewährt und kann aus unserer Sicht auch in diesen Fällen zur Nachahmung empfohlen werden.

Allerdings darf man dabei nicht dem Irrtum erliegen, dass regelkonformes Verhalten eines Verurteilten in der JVA und seine Teilnahme an der Therapie seien a priori Anzeichen fehlender »Gefährlichkeit«. Die Beurteilung der Gefährlichkeit durch den externen Sachverständigen hat sich nicht zuletzt deshalb auf die Erkenntnisse über Art und Umfang früherer Taten und die prädeliktische Persönlichkeit des Verurteilten zu stützen. Es ist durchaus nicht selten, dass gerade diejenigen Täter mit hochproblematischen (z.B. paraphilen) Neigungen sich besonders angepasst verhalten ${ }^{2}$. Insofern bietet die quasi längsschnitthafte zweimalige Begutachtung der gem. § 66a StGB Verurteilten durch ein und denselben Gutachter unverkennbare Vorteile.

\section{Desiderata}

Für eine aufgrund ihrer diagnostischen Funktion an sich wünschenswerte Erhöhung der Begutachtung von Sexualstraftätern im erkennenden Verfahren wäre eine deutliche quantitative und auch qualitative Erhöhung der Gutachterressourcen erforderlich. Unseres Erachtens sollten die Sachverständigen (da sie eben auch Behandlungsindikationen stellen können) idealiter forensisch, sexualmedizinisch und therapeutisch qualifiziert sein. Ein solcher Qualifizierungsbedarf für Sexualmedizin und Forensik besteht im übrigen jetzt schon durch die gestiegene Nachfrage nach Prognosebegutachtungen: Nach unserem Eindruck kommt es aufgrund von Gutachtermangel zur Verzögerung und nicht selten auch zum Ausbleiben der - zu Resozialisierungszwecken und auch für therapeutisch begleitete Erprobungen eigentlich unerlässlichen - vorzeitigen 2/3-Entlassung auf Bewährung. Dringender Qualifikationsbedarf besteht darüber hinaus für ambulante, intra- murale oder in Sozialtherapeutischen Einrichtungen tätige Therapeutinnen und Therapeuten von Sexualstraftätern (Beier et al. 2000). Es ist deshalb zu hoffen, dass die von verschiedenen Fachgesellschaften (unter anderem der DGPPN und der Akademie für Sexualmedizin) entfalteten Initiativen zur Einführung von Curricula und Weiterbildungsnachweisen in Forensischer Psychiatrie (Nedopil \& Sass, 1997; Sass, 2000) und in Sexualmedizin (Vogt et al. 1995; Beier 1999) hier eine Wende zum Besseren bringen.

\section{Literatur}

Beier, K.M. (1999): Sexualmedizin: Berufsbegleitende Fortbildung mit Zertifikat. DT. ÄRZTEBL. 96: A2075-2077.

Beier, K.M./ Bosinski, H.A.G./ Hartmann, U./ Loewit, K. (2001): Sexualmedizin - Grundlagen und Praxis. München: Urban \& Fischer . Beier, K.M.; Hartmann, U.; Bosinski H.A.G. (2000): Bedarfsanalyse zur sexualmedizinischen Versorgung. SEXUOLOGIE 7: 95.

Beier, K.M.; Hinrichs, G. (1995): Psychotherapie mit Straffälligen. Standorte und Thesen zum Verhältnis von Patient - Therapeut Justiz. Stuttgart: Fischer Verlag.

Bosinski, H.A.G. (1997): Sexueller Kindesmißbrauch: Opfer, Täter und Sanktionen. SEXUOLOGIE 4: 27-88.

Nedopil, N./ Sass, H. (1997): Schwerpunkt »Forensische Psychiatrie «? Nervenarzt 68: 529-530

Rotthaus, K.P. (1998): Neue Aufgaben für den Strafvollzug bei der Bekämpfung von Sexualdelikten und anderen gefährlichen Straftaten. NStZ, H. 12: 597-600.

Sass, H. (2000): Zur Musterweiterbildungsordnung: Schwerpunkt »Forensische Psychiatrie«, Weiterbildungscurriculum und Übergangsbestimmungen. Nervenarzt 71: 763-765

Schöch, H. (1999): Zur Offenbarungspflicht der Therapeuten im Justizvollzug gem. § 182 II StVollzG. ZStrVo 5/ 99: 259-266.

Vogt, H.-J./ Loewit, K./ Wille, R./ Beier, K.M./ Bosinski, H.A.G. (1995): Zusatzbezeichnung "Sexualmedizin" - Bedarfsanalyse und Vorschläge für einen Gegenstandskatalog. SEXUOLOGIE 2: 65-89.

Anschrift des Verfassers: Prof. Dr.med. Hartmut A.G. Bosinski; Sexualmedizinische Forschungs- und Beratungsstelle, Universitätsklinikum SchleswigHolstein, Campus Kiel; Arnold-HellerStr. 12; 24105 Kiel;

Email: hagbosi@sexmed.uni-kiel.de

\section{Anmerkungen:}

1 Das »Gesetz zur Bekämpfung von Sexualdelikten und anderen gefährlichen Straftaten « vom Januar 1998 sowie die in seiner Folge vorgenommenen Gesetzesänderungen legen einen Schwerpunkt auch auf die Installation psycho- oder sozialtherapeutischer Maßnahmen zur Behandlung von Sexualstraftätern. Dies wird schon durch die Information des Bundesjustizministeriums (BMJ) zur Einführung des o.g. Gesetzes (BMJ v. 14.11.1997) deutlich, wenn es dort heißt: »Um insbesondere die Gefahr von Wiederholungstaten $\mathrm{zu}$ reduzieren, setzt das Gesetz auf eine Erweiterung der Therapiemöglichkeiten für behandelbare Straftäter im
Strafvollzug. « Das Strafvollzugsgesetz (StVollzG) sieht unter bestimmten Bedingungen regelhaft die Verlegung von Sexualstraftätern (mit Straftaten gem. $\S \S 174$ bis 180 oder 182 StGB) in eine sozialtherapeutische Anstalt vor, die neue Fassung des § 56c StGB ermöglicht nun die gerichtliche Auflage (für die Bewährung oder die Führungsaufsicht) einer psychotherapeutischen Behandlung auch ohne Zustimmung des Betreffenden usw.

2 Schon deshalb verweist die von uns herausgegebene Therapiemeldung auch darauf, dass die Zuordnung des Probanden zu einem bestimmten Therapiestadium keine Einschätzung der Eignung für Lockerungen oder vorzeitige Entlassung darstellt.

\section{Verschärfung des Sexualstrafrechts}

\section{nun Gesetz}

Monika Frommel

Am 19.12 2003 hat der Bundestag ein Gesetz zur Verschärfung des Sexualstrafrechts beschlossen (BGBI I S. 3007). Es gleicht den Strafrahmen des schweren Falls des Missbrauches Widerstandsunfähiger an den der Vergewaltigung (mindestens zwei Jahre) an und verschärft entsprechend auch bei schwerem sexuellen Missbrauch von Kindern die Mindeststrafe (von einem auf zwei Jahre). Reformiert wurden ferner die Anforderungen an Gentests. Einerseits werden sie nun auch bei leichten Sexualstraftaten zugelassen, andererseits beginnt man über bessere Kontrollen nachzudenken, mit begrenztem Erfolg allerdings. Schließlich verschärft dieses Gesetz den Anwendungsbereich der vorbehaltenen Sicherungsverwahrung. Sie kann künftig auch gegen Heranwachsende verhängt werden, bei denen das allgemeine Strafrecht angewandt wird. Mit anderen Worten: wenig Licht und viel Schatten

Keine Anzeigepflicht bei Sexualstraftaten, aber Verschärfung des Strafrahmens bei sexuellem Missbrauch

n NK 2/2003 berichteten wir über die Absichten des Justizministeriums, eine strafbewehrte Anzeigepflicht bei sexuellem Missbrauch und sexueller Nötigung/Vergewaltigung einzuführen. Sie scheiterte am Widerstand der Länder. An der fehlenden Mehrheit gescheitert ist auch der Vorstoß der Opposition zur nachträglichen Sicherungsverwahrung. Es bleibt also beim Kompromiss der vorbehaltenen Sicherungsverwahrung. Diese wird aber auf Heranwachsende, die nach allgemeinem Strafrecht verurteilt worden sind, erweitert. Zwar wird die Zahl der Heranwachsenden, bei denen die Anwendung des JGG verneint worden ist, gering sein aber schon die Tatsache, dass man die vorbehaltene Sicherungsverwah- 